\title{
Uma discussão sobre a eficácia de três métodos geofísicos na investigação de uma área contaminada por hidrocarbonetos
}

\author{
Otávio Coaracy Brasil Gandolfo* - Instituto de Pesquisas Tecnológicas de São Paulo
}

Copyright 2016, SBGf - Sociedade Brasileira de Geofísica

Este texto foi preparado para a apresentação no VII Simpósio Brasileiro de Geofísica, Ouro Preto, 25 a 27 de outubro de 2016. Seu conteúdo foi revisado pelo Comite Técnico do VII SimBGf, mas não necessariamente representa a opinião da SBGf ou de seus associados. E proibida a reprodução total ou parcial deste material para propósitos comerciais sem prévia autorização da SBGf.

\section{Resumo}

Resistivity methods (dipole-dipole resistivity profiling and vertical electrical sounding), ground penetrating radar (GPR) and electromagnetic methods (EM) were all used to characterize a hydrocarbon contaminated site. The anomalous conductive zone, situated in extremely resistive environment, was not well detected by EM method. Also, it was not able to yield the "shadow zone" on GPR profiles. However, very good results were obtained using $2 \mathrm{D}$ resistivity imaging, clearly identifying the conductivity anomaly.

\section{Introdução}

$\mathrm{Na}$ área da geofísica ambiental, quando o objetivo da investigação é a identificação e o mapeamento de anomalias associadas a plumas de contaminantes, já é consolidada a aplicação dos métodos geoelétricos, sendo amplamente utilizados a eletrorresistividade (ER), os métodos eletromagnéticos (EM) e o GPR.

O Manual de Gerenciamento de Áreas Contaminadas da CETESB (Companhia Ambiental do Estado de São Paulo) dedica integralmente um capítulo aos métodos geofísicos, Cap.VI - Investigação confirmatória (CETESB, 2015). Estes três métodos, além da magnetometria, são aqueles recomendados para serem utilizados nos estudos ambientais.

Resultados satisfatórios geralmente são alcançados no mapeamento de anomalias eletricamente condutivas associadas a plumas de contaminação por compostos inorgânicos como, por exemplo, o percolado de aterros sanitários e lixões (Lago et al., 2006).

Entretanto, plumas de contaminação por compostos orgânicos e de hidrocarbonetos são mais difíceis de serem mapeadas por estes métodos geofísicos. Além do mais, possuem um comportamento complexo, ambíguo e que pode variar com o decorrer do tempo, devido a uma variedade de processos como volatilização, oxidação, biodegradação, dentre outros (Olhoef, 1992; Sauck, 1998).

Os hidrocarbonetos são caracterizados por altos valores de resistividade elétrica e baixa permissividade dielétrica (fato verificado em experimentos controlados e de laboratório). Em uma área contaminada, todavia, apresentam um comportamento diferenciado devido às situações dinâmicas que são comumente encontradas "in situ".

Ao longo do tempo pode ocorrer o aparecimento de anomalias de condutividade elétrica associadas a plumas de contaminantes orgânicos, em decorrência de processos biogeoquímicos em subsuperfície que se desenvolvem no meio e no entorno da área contaminada por estes produtos (Atekwana et al., 2000).

Todavia, além da baixa concentração que geralmente apresentam, os contrastes entre estes contaminantes e o material litológico hospedeiro podem ser de pequena magnitude, o que dificulta a sua detecção e o mapeamento de sua extensão (Olhoeft, 1992).

Serão aqui apresentados e discutidos os resultados de um levantamento geofísico, onde foram utilizados três métodos geofísicos, ER, EM e GPR, em uma área onde foi detectada a presença de hidrocarbonetos (HC), indicando uma contaminação do solo e da água subterrânea por estes compostos químicos.

Nesta área, durante uma campanha de caracterização geotécnica do subsolo voltada para as fundações de novas obras, foram realizadas diversas sondagens mecânicas à percussão (SPT), sendo que uma delas, acidentalmente, detectou a presença da contaminação em subsuperfície.

\section{Descrição geológica e hidrogeológica da área}

A área investigada encontra-se inserida na Bacia Sedimentar de Taubaté. Localmente, observa-se a uma camada superior de aterro (aproximadamente 2 metros de espessura), seguida por pacotes arenosos com granulometria fina a média (permeabilidade com valores $\mathrm{da}$ ordem de grandeza entre $10^{-3}$ e $10^{-4} \mathrm{~cm} / \mathrm{s}$ ), intercalados com estratos e lentes de argila siltosa, ambos de idade recente (Quaternário).

O nível d’água local encontrava-se a 13 metros de profundidade na ocasião em que o levantamento geofísico foi realizado.

\section{Levantamentos de campo}

Os dados de campo, empregando os três métodos, foram adquiridos em uma linha com 200 metros de extensão, ao longo da qual existiam dois pontos de sondagens, SP-09 e SP-10.

$\mathrm{Na}$ sondagem SP-09 (posição 130m) foi detectada uma contaminação por $\mathrm{HC}$, enquanto que na SP-10 (posição $70 \mathrm{~m})$, não foi detectado nenhum indício de contaminantes em subsuperfície. 
Na Figura 1 é apresentada uma foto com uma amostra de solo coletada nas proximidades da sondagem SP-09, nos trabalhos de diagnóstico desta área contaminada.

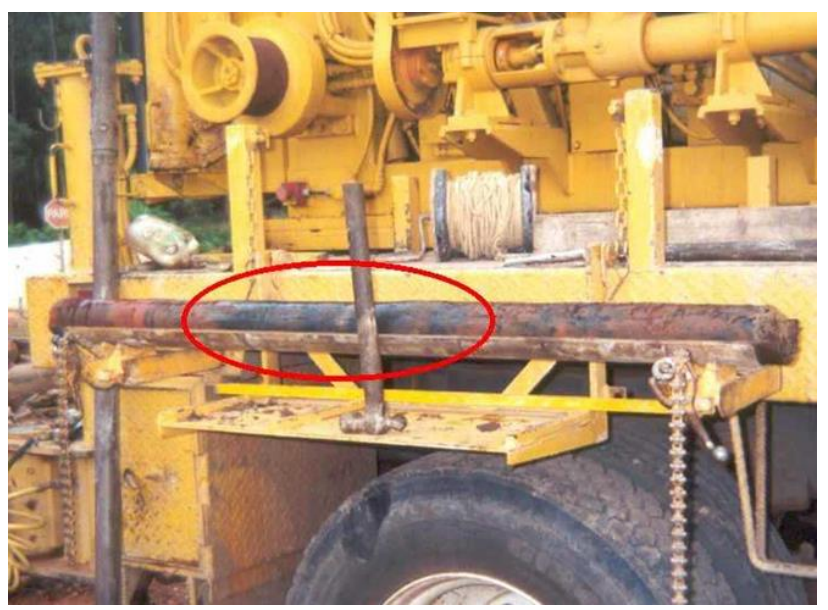

Figura 1 - Amostra de solo contaminada por HC.

Os parâmetros de campo e os equipamentos utilizados para a aquisição dos dados serão descrito a seguir.

\subsection{Eletrorresistividade (ER)}

Foi realizado um caminhamento elétrico com o arranjo dipolo-dipolo, espaçamento entre eletrodos igual a $10 \mathrm{~m} \mathrm{e}$ 4 níveis de investigação em profundidade.

$\mathrm{Na}$ posição $130 \mathrm{~m}$ do perfil, no local da ocorrência da contaminação, foi realizada uma sondagem elétrica vertical (SEV), com máxima abertura de eletrodos igual a $100 \mathrm{~m}$. O equipamento utilizado foi um Tectrol, modelo TDC 1000/24.

\subsection{Eletromagnético (EM)}

O caminhamento eletromagnético foi realizado utilizando o equipamento EM-34, com espaçamento entre as bobinas transmissora e receptora iguais a $10 \mathrm{~m}$ e $20 \mathrm{~m}$, nos modos de medida "dipolo vertical" e "horizontal", possibilitando a investigação em três níveis de profundidade: até $7,5 \mathrm{~m}$, até $15 \mathrm{~m}$ e até $30 \mathrm{~m}$ (McNeill, 1980).

Foram efetuadas medidas a cada $10 \mathrm{~m}$ ao longo do perfil. $\mathrm{Na}$ área do levantamento não existiam interferências causadoras de ruídos eletromagnéticos.

\subsection{GPR}

O levantamento GPR foi realizado utilizando um equipamento da RAMAC (Malä Geoscience), com uma antena não blindada de frequência central igual a $50 \mathrm{MHz}$. O espaçamento entre traços foi de $0,5 \mathrm{~m}$.

Foi também realizado um ensaio CMP para obtenção "in situ" da velocidade de propagação da onda eletromagnética, parâmetro que foi utilizado no processamento dos dados para a conversão tempoprofundidade.
A escolha da antena de $50 \mathrm{MHz}$ teve por finalidade alcançar maiores profundidades de investigação (em torno de $13 \mathrm{~m}$ ), procurando "imagear" toda a camada não saturada do terreno.

\section{Discussão dos resultados}

A curva de resistividade aparente $\left(\rho_{a}\right)$, obtida da SEV, e o respectivo modelo geoelétrico interpretado, são apresentados na Figura 2.

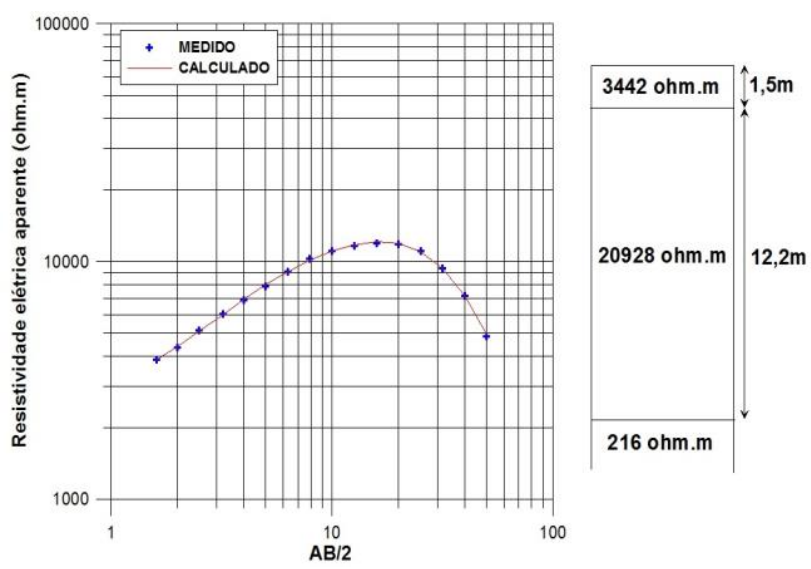

Figura 2 - Resultados da SEV (curva de resistividade elétrica aparente e o modelo geoelétrico interpretado).

O modelo de 3 camadas, no padrão $\rho_{1}<\rho_{2}>\rho_{3}$ (curva tipo K), foi assim interpretado: a) $1^{\text {a }}$ camada, $\rho_{1}=3442$ ohm.m, aterro com 1,5 metros de espessura; b) $2^{\text {a }}$ camada, $\rho_{2}=20928$ ohm.m, solo arenoso seco, com 12,2 metros de espessura; c) $3^{\text {a }}$ camada, $\rho_{3}=216$ ohm.m, zona saturada do terreno.

Os dados do caminhamento elétrico, apresentados na forma de pseudo-seção, confirmam a ocorrência de valores muito elevados de $\rho_{\mathrm{a}}$ medidos ao longo de toda a seção 2D investigada (Figura 3). Neste ambiente altamente resistivo foi identificada uma zona anômala nas proximidades da sondagem SP-09, com valores de $\rho_{a}$ consideravelmente menores em relação ao meio circundante.

A seção modelada foi obtida por processo de inversão dos dados, utilizando o software RES2DINV (Geotomo Software). Devido à grande variação dos valores de $\rho_{a}$, visando uma melhoria na qualidade da seção modelada, foram utilizados os logaritmos das resistividades para inversão dos dados. Isto não representa prejuízo algum no resultado final, uma vez que a investigação geofísica está interessada no contraste dos parâmetros e não especificamente nos valores da grandeza física medida.

$\mathrm{Na}$ seção modelada de resistividade (Figura 4) observase a presença de uma camada menos resistiva na superfície, em tonalidades na cor verde, que corresponde ao aterro. Abaixo desta, encontra-se um espesso pacote com elevados valores de resistividade elétrica (nas cores avermelhadas), correspondente à zona vadosa composta pelos sedimentos finos predominantemente arenosos. 
A posição da anomalia de condutividade ficou bem definida em uma estreita faixa em torno da SP-09 (em tonalidades azuis), abrangendo boa parte da zona vadosa do terreno. Sauck et al. (1998) mostraram que a ER foi o método que forneceu o melhor resultado na detecção de uma pluma condutiva associada à contaminação por $\mathrm{HC}$ em uma condição hidrogeológica semelhante. Atekwana et al. (2002) discutem a possibilidade da ocorrência de processos de biodegradação na zona vadosa do terreno.

Os resultados do levantamento EM são apresentados na forma de perfis de condutividade elétrica aparente $\left(\sigma_{\mathrm{ap}}\right)$, nos três níveis de investigação (Figura 5). Verifica-se o aumento de $\sigma_{\mathrm{ap}}$ com a profundidade. Nos dois níveis mais rasos (até $7,5 \mathrm{~m}$ e até $15 \mathrm{~m}$ ) é pequena a influência da zona saturada, pois o N.A. encontrava-se a $13 \mathrm{~m}$ de profundidade. Os valores médios de $\sigma_{\mathrm{ap}}$ para estes dois níveis são iguais a $2,5 \mathrm{mS} / \mathrm{m}$ e $4,6 \mathrm{mS} / \mathrm{m}$, respectivamente. No nível mais profundo (investigação até $30 \mathrm{~m}$ ), é evidente a influência da porção saturada do solo, com o valor médio de $\sigma_{\mathrm{ap}}$ igual a $16,7 \mathrm{mS} / \mathrm{m}$.

Um pequeno pico de condutividade pode ser observado no perfil correspondente à profundidade de até $15 \mathrm{~m}$ (em vermelho na Figura 5), porém não muito significativo e que, certamente, passaria despercebido não fosse 0 conhecimento de que no local existia a contaminação.

Tomando-se os valores de $\sigma_{\mathrm{ap}}$ calculados a partir do inverso das $\rho_{a}$ obtidas do caminhamento elétrico, observa-se que os contrastes de $\sigma_{\mathrm{ap}}$ não apresentaram uma magnitude suficiente para serem identificados no levantamento eletromagnético com 0 equipamento EM-34. Nash et al. (1997) mostram que os resultados apresentados pelo EM na investigação de uma área contaminada por HC também não apresentaram uma boa resposta no mapeamento da zona condutiva.

O ambiente altamente resistivo, constatado pelos dados de ER (SEV e caminhamento elétrico) contribuiu para a obtenção de uma seção de boa qualidade e para uma boa penetração do sinal do GPR em profundidade.

Já é de amplo conhecimento que o melhor desempenho do GPR está em litologias que apresentem altos valores de resistividade elétrica, onde a expectativa de penetração do sinal pode ser superior a 10 metros de profundidade (Figura 6).

O registro do ensaio CMP, para obtenção da velocidade de propagação da onda no meio, é apresentado na (Figura 7). O valor determinado, $\mathrm{v}=0,1 \mathrm{~m} / \mathrm{ns}$, foi utilizado para a conversão tempo-profundidade da seção GPR apresentada na Figura 8.

No local com a presença da contaminação por $\mathrm{HC}$ ( $X=130 \mathrm{~m}, \mathrm{SP}-09$ ), onde a anomalia condutiva ficou bem caracterizada pelos dados de ER (na pseudo-seção e na seção modelada), não foi observada nenhuma alteração no padrão do sinal GPR. Especificamente, não foi verificada nenhuma atenuação da onda eletromagnética, gerando a zona de sombra ("shadow zone"), comportamento esperado em meios eletricamente condutivos.

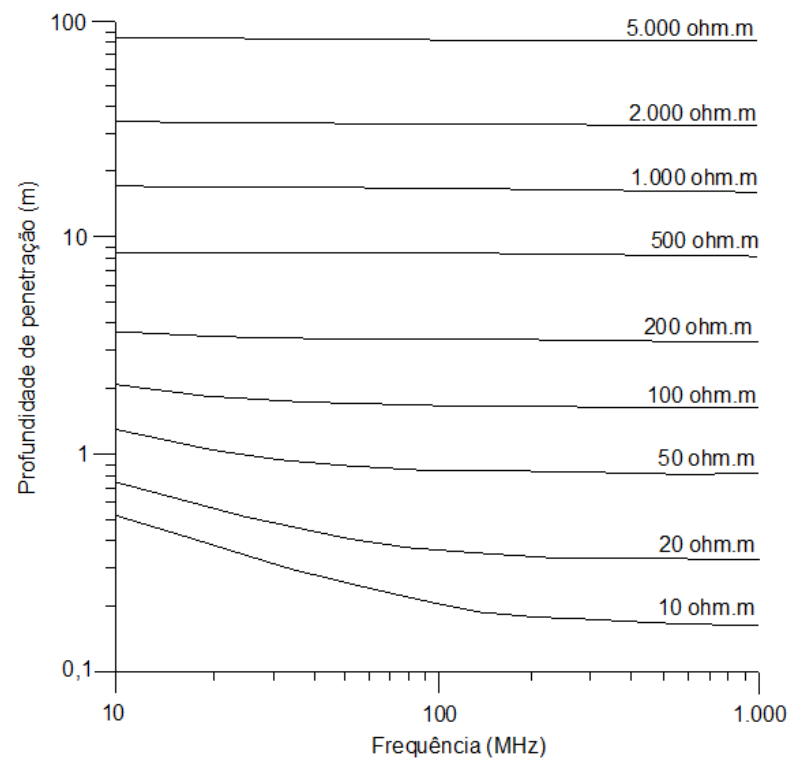

Figura 6 - Relação entre profundidade de penetração, frequência e resistividade elétrica para o método GPR (modificado de Parasnis, 1977).

A Figura 9 mostra um bom exemplo de uma aplicação, com sucesso, do método GPR na detecção e mapeamento de chorume proveniente de um aterro sanitário. Observa-se que a "shadow zone" interpretada por Davis \& Annan (1989) está limitada pela linha que representa os valores de $\sigma$ maiores que $10 \mathrm{mS} / \mathrm{m}$. Ou seja, a atenuação do sinal ocorreu nas regiões em subsuperfície onde $\rho_{a}$ são menores que 100 ohm.m.

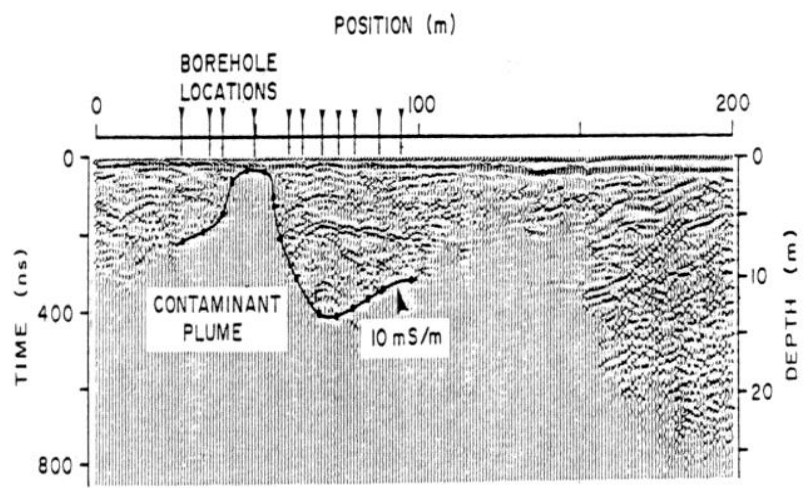

Figura 9 - Seção GPR obtida no entorno de uma área de aterro sanitário (Davis \& Annan, 1989).

Neste estudo de caso, onde a anomalia de condutividade apresentou valores de $\rho_{\mathrm{a}}$ da ordem de grandeza de poucos milhares de ohm.m (de 1.000 a 3.500ohm.m, aproximadamente), a magnitude destes não causou a atenuação do sinal do GPR e, consequentemente, o aparecimento da "shadow zone".

Entretanto, esta anomalia foi muito bem identificada pela ER devido ao grande contraste existente entre ela e o meio encaixante, constituído por materiais com valores de resistividade elétrica ainda mais elevados. 


\section{Conclusões}

Em um ambiente geoelétrico com a presença de materiais extremamente resistivos (sedimentos arenosos finos a médios e com profundidade do nível d'água igual a 13 metros), o método da eletrorresistividade (ER) foi o que apresentou resultado satisfatório na identificação da anomalia de condutividade associada a uma pluma de contaminação por HC. A anomalia condutiva foi identificada tanto na camada saturada como na zona vadosa do terreno.

A magnitude do contraste do parâmetro físico investigado ( $\sigma_{\mathrm{ap}}$ ou $\left.\rho_{\mathrm{ap}}\right)$, bem identificado pela $\mathrm{ER}$, não foi suficiente para produzir anomalias detectáveis para os métodos EM e GPR.

\section{Referências bibliográficas}

Atekwana, E.A., Sauck, W.A.; Werkema, D.D. 2000. Investigations of geoelectrical signatures at a hydrocarbon contaminated site. Journal of Applied Geophysics, n. 44, v.2, p.167-180.

Atekwana, E.A., Sauck, W.A., Abdel Aal, G.Z., Werkema Jr, D.D. 2002. Geophysical investigation of vadose zone conductivity anomalies at a hydrocarbon contaminated site: implications for the assessment of intrinsic bioremediation. Journal of Environmental \& Engineering Geophysics, v. 7, n.3, p. 103-110.

CETESB. 2015. Manual de Gerenciamento de Áreas Contaminadas. Capítulo VI - Investigação confirmatória (6200 - Métodos geofísicos). Disponível em:

http://areascontaminadas.cetesb.sp.gov.br/wpcontent/uploads/sites/45/2013/11/6200.pdf

Davis, J.L., Annan, A.P. 1989. Ground-penetrating radar for high-resolution mapping of soil and rock stratigraphy. Geophysical Prospecting, v.37, n.5, p.531-551.

Lago, A., Elis, V.R., Giacheti, H.L. 2006. Aplicação integrada de métodos geofísicos em uma área de disposição de resíduos sólidos urbanos em BauruSP. Revista Brasileira de Geofísica, v. 24, n. 3, p. 357374.

McNeill, J.D. 1980. Electromagnetic Terrain Conductivity Measurement at Low Induction Number. Geonics Technical Note, TN-6, 15p.

Nash, M.S., Atekwana, E., Sauck, W. A. 1997. Geophysical investigation of anomalous conductivity at a hydrocarbon contaminated site. In: Proceedings of the Symposium on the Application of Geophysics to Engineering and Environmental Problems (SAGEEP'97), Reno, NV, p.675-683.
Olhoeft, G.R. 1992. Geophysical detection of hydrocarbon and organic chemical contamination. In: Proceedings of the Symposium on the Application of Geophysics to Engineering and Environmental Problems (SAGEEP'92), Oakbrook, IL, p.587-595.

Parasnis, D.S. 1997. Principles of applied geophysics. 5ed. London: Chapman and Hall, 429p.

Sauck, W.A. 1998. A conceptual model for the geoelectrical response of LNAPL plumes in granular sediments. In: Proceedings of the Symposium on the Application of Geophysics to Engineering and Environmental Problems (SAGEEP'98), Chicago, IL, p.805-817.

Sauck, W.A., Atekwana, E.A, Nash, M.S. 1998. High conductivities associated with an LNAPL plume imaged by integrated geophysical techniques. Journal of Environmental and Engineering Geophysics, v.2, p.203212. 


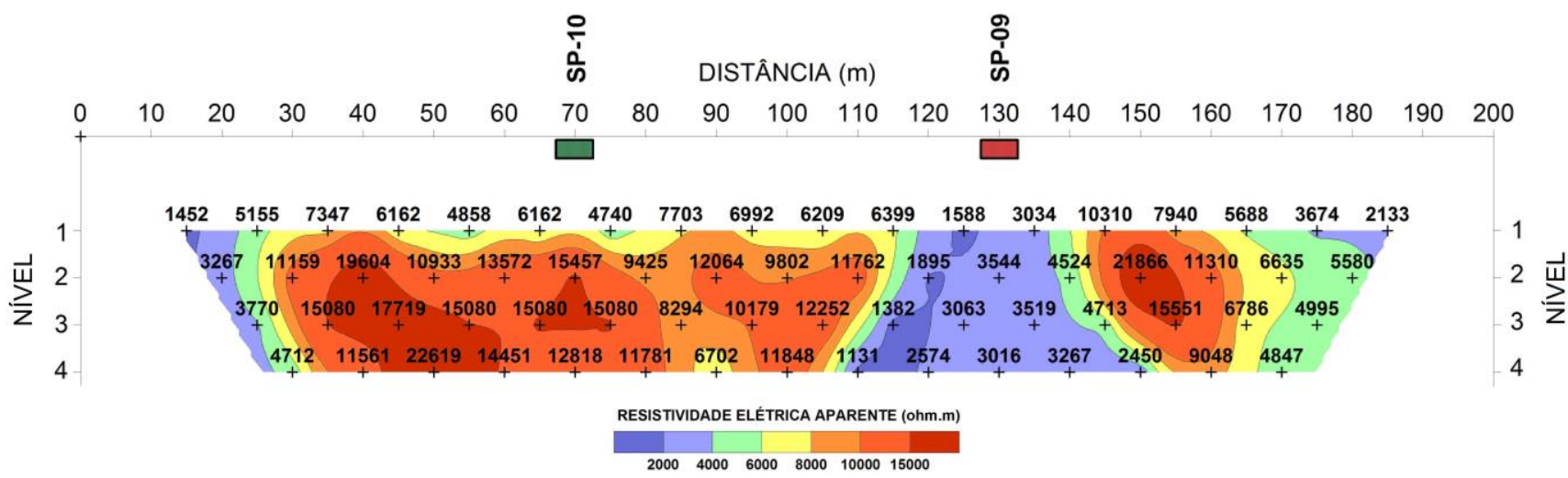

Figura 3 - Pseudo-seção de resistividade elétrica aparente.

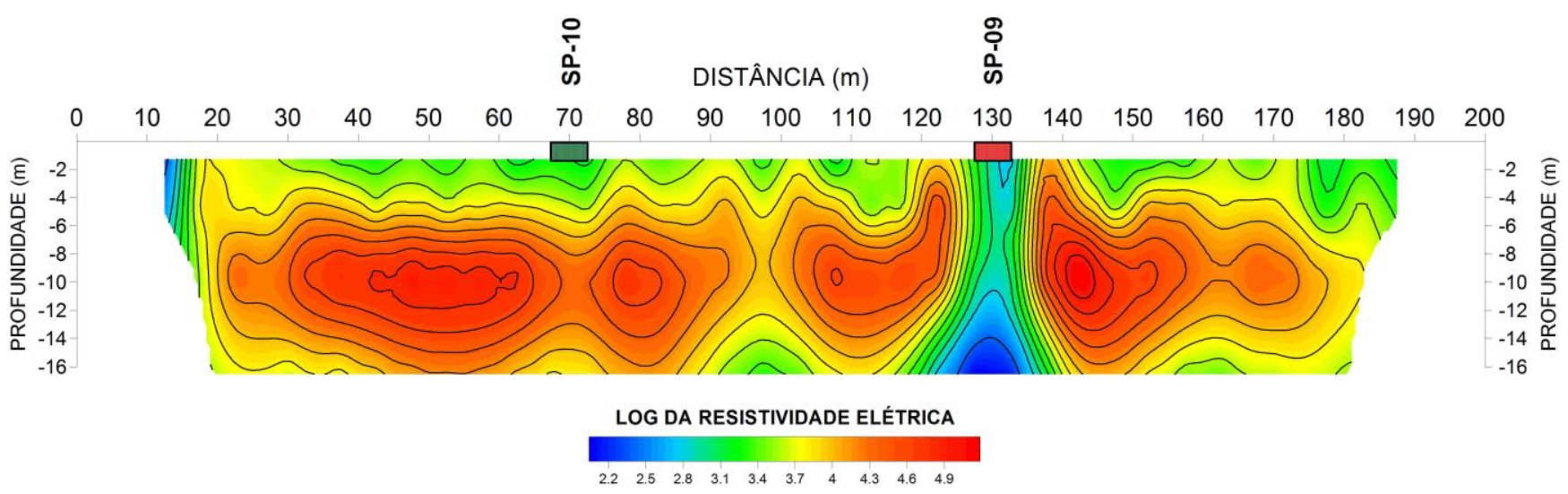

Figura 4 - Seção modelada de resistividade elétrica (5 iterações, erro de RMS igual a 1,2\%).

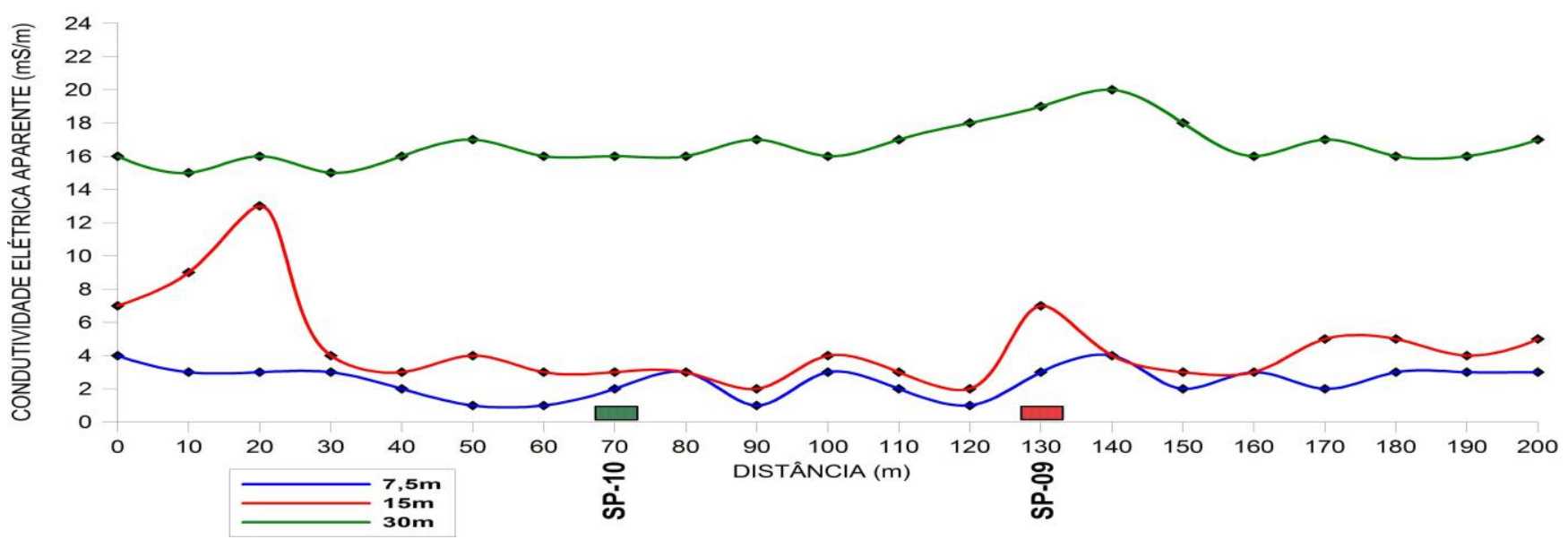

Figura 5 - Perfis de condutividade elétrica aparente

SP-10 - NÃO IDENTIFICOU CONTAMINAÇÃO POR HC SP-09 - IDENTIFICOU CONTAMINAÇÃO POR HC 


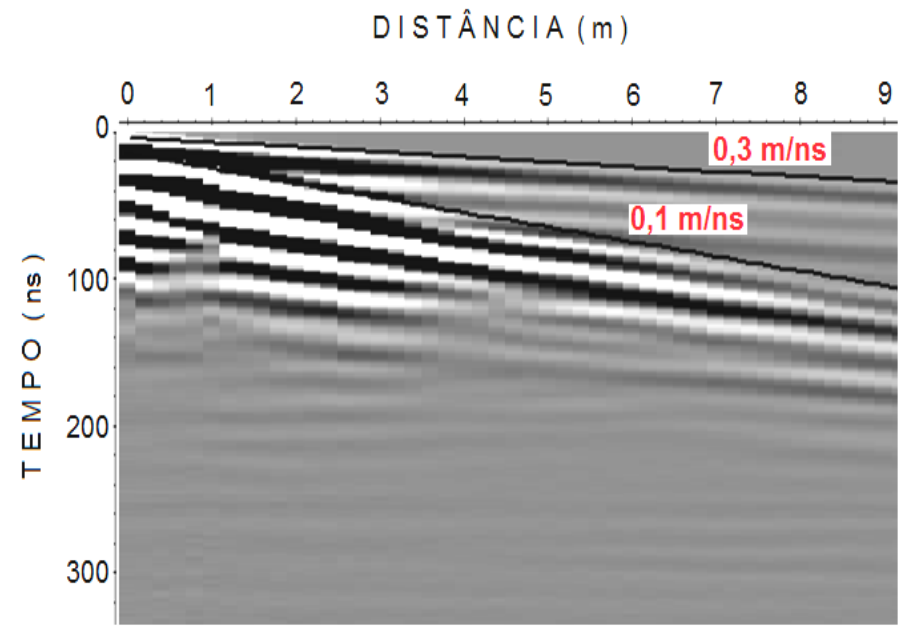

Figura 7 - Registro do ensaio CMP para obtenção das velocidades.

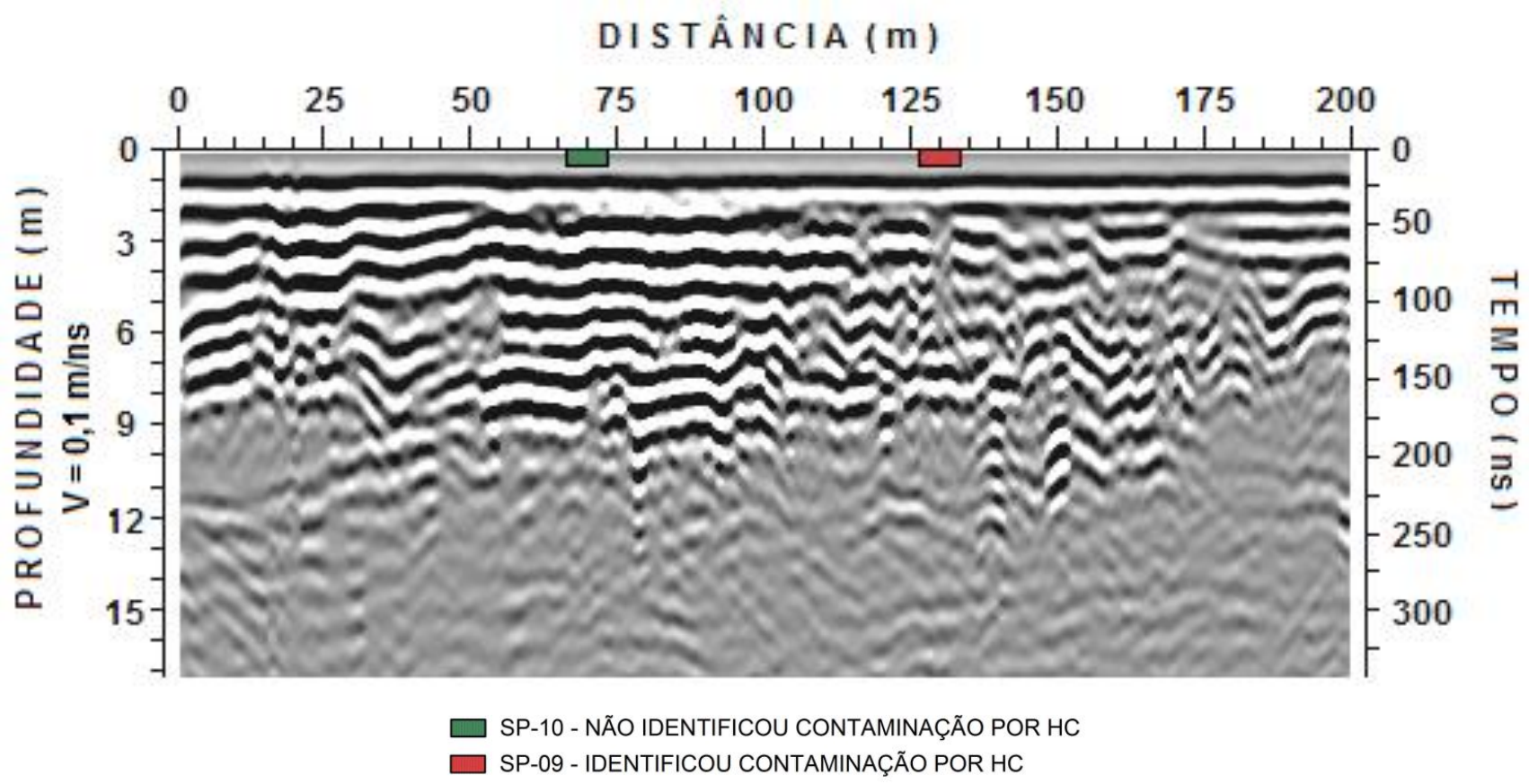

Figura 8 - Seção GPR obtida em ambiente eletricamente resistivo com uma antena de $50 \mathrm{MHz}$. A presença da anomalia condutiva não foi detectada na posição $130 \mathrm{~m}$. 